\title{
Button Batteries as Missed Foreign Bodies in Children
}

\author{
Body $\mathrm{S}^{1}$, Fraser $\mathrm{L}^{2}$, Geyer $\mathrm{M}^{3}$, Burgess $\mathrm{A}^{1}$ and Ismail-Koch $\mathrm{H}^{1 *}$ \\ ${ }^{1}$ Department of Otorhinolaryngology, University Hospital Southampton, UK \\ ${ }^{2}$ Department of Otorhinolaryngology, Oxford University Hospitals, UK \\ ${ }^{3}$ Department of Otorhinolaryngology, Salisbury District Hospital, UK
}

Case Report

Volume 2 Issue 2

Received Date: October 07, 2017

Published Date: October 12, 2017

DOI: $10.23880 /$ ooaj-16000157

*Corresponding author: Hasnaa Ismail-Koch, Department of Otorhinolaryngology, Southampton Children's Hospital, University Hospital Southampton, Tremona Road, Southampton, UK, E-mail: hasnaa@doctors.org.uk

\section{Abstract}

The dangers of button battery ingestion are well documented. Button batteries may also be inserted into the ears and nose. In many cases of foreign body insertion the nature of the foreign body may be unclear. We describe two cases where foreign bodies of metallic appearance were noted in the ear and nose respectively with no prior history of button battery placement. Button batteries, which had caused significant tissue damage, were discovered in both cases. When there is diagnostic uncertainty regarding the precise nature of a metallic looking foreign body in the ears, nose or upper aerodigestive tract there should be a high index of suspicion of the possibility of a button battery and urgent removal undertaken.

Keywords: Button batteries; Foreign bodies; Upper aerodigestive

\section{Introduction}

Foreign bodies in the nose, ear and pharynx are common presentations with the most concerning items being button batteries due to their destructive tendencies. In 2014, NHS England issued a patient safety alert regarding the risks of delay in recognising button battery ingestion [1]. With the increasing use of electronic toys and other household gadgets, it follows that the incidence of harm from button battery ingestion [2] and insertion into the ears and nose is increasing. It may be difficult to ascertain the nature of foreign body in a child and therefore delayed diagnosis is not uncommon and may present with long term complications. Metallic looking foreign bodies may be button batteries, despite no clear history of insertion. Plain X-ray films in anteroposterior and lateral views if performed can often help diagnosis as a distinctive bilaminar structure appearing as a "double ring" or "halo" appearance will be seen. The two cases below highlight the damage caused by delayed removal of button batteries due to the unknown nature of the metallic looking foreign bodies.

\section{Case Reports}

\section{Case 1}

A 5 year old boy presented on day 2 following insertion of a metallic foreign body in the nose. It was not possible to remove the foreign body whilst awake and an examination under general anaesthesia was required. A small button battery was removed from the right nasal cavity and surgical debridement of the damaged tissue performed (Figure 1). Antibiotics and nasal douches were administered postoperatively. The button battery resulted in a septal perforation and the child remains under follow up. 


\section{Otolaryngology Open Access Journal}

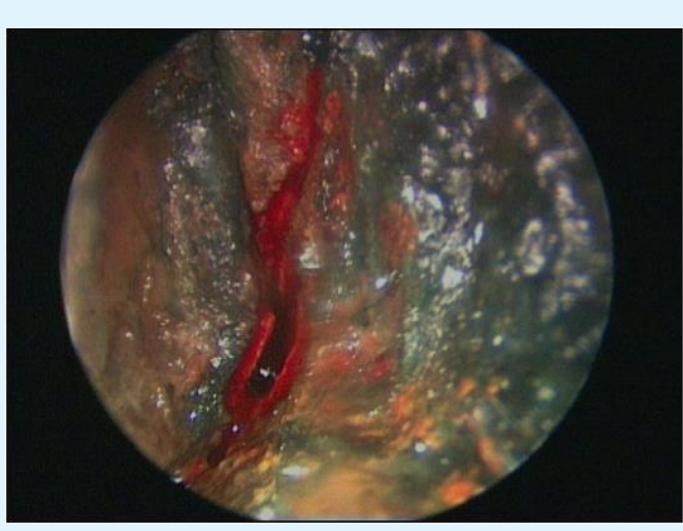

Figure 1: Endoscopic view of the right nasal cavity following removal of a button battery, showing damage to the inferior turbinate, middle turbinate and septum.

\section{Case 2}

An 8 year old boy with known attention deficit hyperactivity disorder (ADHD) presented with an unknown foreign body of metallic appearance in the right ear. It was not possible to remove the foreign body whilst he was awake. Therefore he was taken to theatre on day 2 for removal under general anaesthesia. Intraoperatively a button battery was identified which had eroded the tympanic membrane and could be seen in the middle ear. Removal necessitated a post-auricular approach. Damage caused by the button battery resulted in stenosis of the external auditory canal with mild conductive hearing loss (Figure 2). The perforation healed with no intervention.

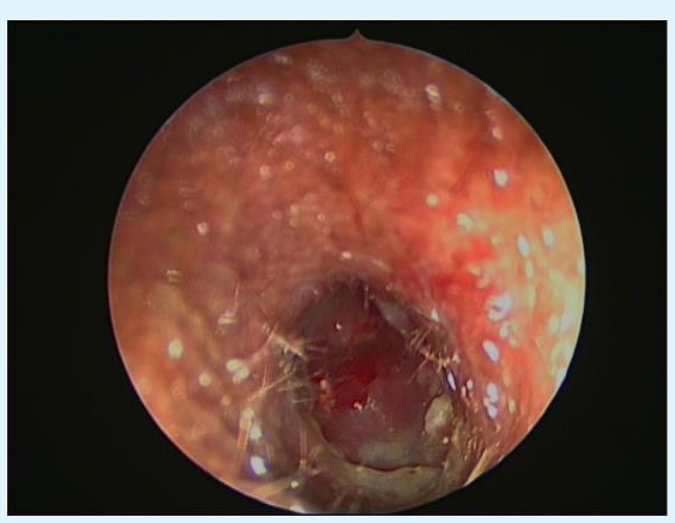

Figure 2: Endoscopic view of the right external auditory canal following removal of a button battery, showing stenosis of the external auditory canal.

\section{Discussion}

The majority of button batteries today are of alkaline varieties, which cause liquefaction necrosis. The mechanism of injury entails: a) Leakage of the battery contents with direct corrosive damage

b) Direct electrical currents on the mucosa

c) Pressure necrosis form prolonged local pressure

d) Local toxic effects due to absorption of substances

Button battery ingestion, especially in small children has been shown to cause significant injury rapidly, leading to long-term morbidity and possible death [3].

Nasal button battery impaction may produce mucosal damage including turbinate and septal ulceration in just 3-6 hours with necrosis of the inferior turbinate occurring at 24 hours [4].

\section{Conclusion}

There should be a high index of suspicion when metallic foreign bodies are seen in the ears or nose of children. The aim should be to avoid misdiagnosis and delayed treatment. The potential presence of a button battery in the ear, nose and/or upper aerodigestive tract represents an otorhinolaryngological emergency requiring immediate removal of the foreign body. It may be possible to remove a foreign body in a cooperative child in the emergency clinic, however often a general anaesthetic is required for removal and debridement. Parental and provider education remain the most important factors to prevent button battery injury. Collaboration between healthcare professionals and industry is essential to find a safer solution to this ongoing hazard [3].

\section{References}

1. NHS England (2014) Risk of Death and Serious Harm from Delays in Recognising and Treating Ingestion of Button Batteries.

2. Litovitz T, Whitaker N, Clark L, Nicole CW, Melinda M (2010) Emerging Battery-Ingestion Hazard: Clinical Implications. Pediatrics 125(6): 1168-1177.

3. Eliason MJ, Ricca RL, Gallagher TQ (2017) Button battery ingestion in children. Curr Opin Otolaryngol Head Neck Surg.

4. Alvi A, Bereliani A, Zahtz GD (1997) Minature disc battery in nose: a dangerous foreign body. Clinical Paediatr 36(7): 427-429.

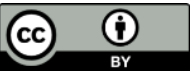

